\title{
Comparison of bispectral index scores from the standard frontal sensor position with those from an alternative mandibular position
}

\author{
Shin Young Lee, Young Sung Kim, Byung Gun Lim, Heezoo Kim, Myoung-Hoon Kong, and Il-Ok Lee \\ Department of Anesthesiology and Pain Medicine, Korea University Guro Hospital, Seoul, Korea
}

Background: The standard bifrontal application of the bispectral index (BIS) sensor interferes with the operative field in neurosurgery and plastic surgery. The aim of this study was to compare the standard frontal BIS sensor position with an alternative position across the mandible.

Methods: Two BIS ${ }^{\mathrm{TM}}$ Quatro sensors (Aspect Medical Systems, Newton, MA, USA) mounted on the frontal and mandibular regions were connected to BIS Vista ${ }^{\mathrm{TM}}$ monitors on each patient during general anesthesia. Data from each position were collected at awake, loss of consciousness, intubation, incision, every 30 minutes during the intraoperative period and emergence. These data were compared using Bland-Altman and scatter plot analyses.

Results: Scatter plot analysis revealed a significant correlation between BIS values of frontal and mandibular positions $(\mathrm{R}=0.869, \mathrm{P}=0.000)$, except during emergence $(\mathrm{R}=0.253, \mathrm{P}=0.077)$. Bland-Altman analysis revealed a negative bias of 3.2 with a limit of agreement of $16.5 /-22.9$, in which $3.7 \%$ of the values were outside of the limit of agreement. Additional values included $-2.9(14.1 /-8.3)$ while patients were awake, $-21.7(14.9 /-58.3)$ at loss of consciousness, -1.8 (9.0/-12.5) during maintenance, and $-1.9(14.9 /-18.8)$ during emergence.

Conclusions: Overall, BIS values do not agree between the standard frontal position and an alternative mandibular position. However, during the anesthesia maintenance period, the mandibular position can be availably used as an alternative position if the operative field renders the standard frontal position unavailable. (Korean J Anesthesiol 2014; 66: 267-273)

Key Words: Bispectral index monitors, Electroencephalography, General anesthesia, Mandible.

Received: August 14, 2013. Revised: 1st, September 16, 2013; 2nd, October 7, 2013. Accepted: October 10, 2013.

Corresponding author: Il-Ok Lee, M.D., Ph.D., Department of Anesthesiology and Pain Medicine, Korea University Guro Hospital, 148, Gurodong-ro, Guro-gu, Seoul 152-703, Korea. Tel: 82-2-2626-1437, Fax: 82-2-2626-1438, E-mail: iloklee@korea.ac.kr

(c) This is an open-access article distributed under the terms of the Creative Commons Attribution Non-Commercial License (http:// creativecommons.org/licenses/by-nc/3.0/), which permits unrestricted non-commercial use, distribution, and reproduction in any medium, provided the original work is properly cited. 


\section{Introduction}

Approved by the Food and Drug Administration in 1996, the bispectral index (BIS) is the most widely used noninvasive method to estimate level of consciousness [1,2]. The BIS is a complex electroencephalography (EEG) parameter that converts raw EEG data from the frontal cortex into a single number between 100 (fully awake) and 0 (isoelectric EEG) [3].

The BIS sensor is a single adhesive strip placed across the forehead. This position can be problematic in certain surgical procedures involving a fronto-temporal approach, such as those used in plastic surgery and neurosurgery. Although several alternative sensor positions have been examined in previous studies, there remains significant disagreement over their reliability. In one study, occipital placement had a strong relationship $\left(\mathrm{r}^{2}=0.961\right)$ with standard frontal placement during general anesthesia for neurosurgery [4]. In contrast, other studies found poor agreement between occipital and frontal placement with a discrepancy of over \pm 10 BIS units [5,6]. A third study found BIS values with nasal placement had adequate agreement with standard frontal placement [6].

We hypothesized that BIS values from mandibular sensor placement would be significantly correlated with those from the standard frontal position. To test this hypothesis, we compared BIS values between mandibular and frontal sensor positions in individual patients at different stages of anesthesia.

\section{Materials and Methods}

We enrolled 58 patients scheduled for various surgical procedures not involving the head or neck requiring routine general

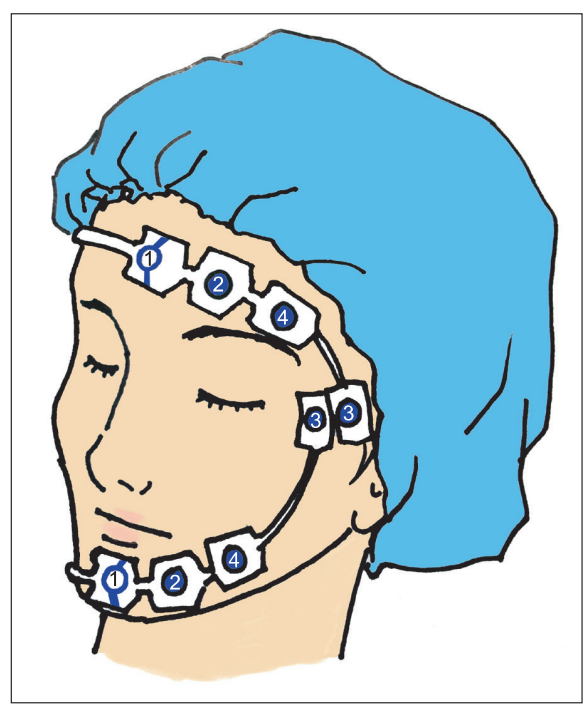

Fig. 1. Location of bispectral index (BIS) sensors. anesthesia. All participants provided written informed consent. Participants were between 18 and 75 years of age and had an American Society of Anesthesiologists (ASA) of I-II. We excluded patients with a history of disabling central nervous or cerebrovascular disease, those currently taking central nervous system active drugs or psychiatric medication, and those who had previously undergone neurosurgery. The Institutional Review Board approved this study.

Before the induction of anesthesia, we applied 2 BIS sensors (BIS ${ }^{\mathrm{TM}}$ Quatro Sensors, Aspect Medical Systems, Newton, MA, USA) to each patient, 1 across the forehead and 1 along the mandible, and attached each sensor to an each BIS monitor (BIS-Vista ${ }^{\mathrm{TM}}$ monitors, Aspect Medical Systems, Newton, MA, USA). Frontal sensors were applied with circle 1 at the center of the forehead; circle 2, $2.8 \mathrm{~cm}$ lateral to circle 1; and circle 3 on either temporal area between the corner of the eye and the hairline. Mandibular sensors were applied to the same side of the face, with circle 1 at the center of the mandible, circle 4 adjacent to the lateral side of lip, and circle 3 at the ipsilateral temporal area (Fig. 1). Anesthesia was induced with $2 \mathrm{mg} / \mathrm{kg}$ of propofol and $0.7 \mathrm{mg} / \mathrm{kg}$ of rocuronium was administered as a muscle relaxant. Then Anesthesia was maintained with sevoflurane or desflurane in $50 \% \mathrm{~N}_{2} \mathrm{O}$. Minimum alveolar concentration range for the inhaled anesthetics was $0.8-1.5$. At the completion of surgery, $10 \mathrm{mg}$ of pyridostigmine with $0.4 \mathrm{mg}$ of glycopyrrolate was given to antagonize any residual neuromuscular block. From each BIS monitor, we collected 3 pieces of data at each of 6 times points: BIS score, signal quality index (SQI), and electromyography (EMG) score before the induction of anesthesia (awake), at loss of the eyelash reflex (LOC), after intubation (intubation), after the first surgical incision (incision), every 30 minutes during the intraoperative period (maintenance), and at spontaneous eye opening upon emergence from anesthesia (emergence). Data were downloaded from BIS monitors after each procedure using a USB port for subsequent analysis. Sudden, high EMG scores were identified as artifacts and the associated BIS values were eliminated in data analysis. During induction, patients were instructed to keep their faces relaxed (eyes closed, mouth closed,

Table 1. Patient Demographics

\begin{tabular}{lc}
\hline \multicolumn{1}{l}{ Variable } \\
\hline Age $(\mathrm{yr})$ & $46.25 \pm 15.36$ \\
Gender $(\mathrm{F} / \mathrm{M})$ & $27 / 31$ \\
ASA $(\mathrm{I} / \mathrm{II})$ & $32 / 26$ \\
Height $(\mathrm{cm})$ & $165.49 \pm 8.17$ \\
Weight $(\mathrm{kg})$ & $64.80 \pm 11.53$ \\
BMI $\left(\mathrm{kg} / \mathrm{m}^{2}\right)$ & $23.49 \pm 3.05$
\end{tabular}

$\mathrm{N}=58$. Data are presented as mean $\pm \mathrm{SD}$ for age, height, weight, and BMI, and as actual number of patients for gender. ASA: American Society of Anesthesiologists physical status classification, BMI: body mass index. 
no facial expressions). In our previous pilot study, we found that the frontal BIS scores fell more quickly than mandibular scores during induction. Therefore, we recorded the difference in time required for the BIS value to reach its lowest level between sensor positions during induction. We complied data from 10 pilot patients in whom the mean BIS values during anesthesia maintenance were $50.1 \pm 10.5$ (frontal position) and $54.2 \pm 5.1$ (mandibular position). Given this preliminary data, to achieve an alpha of 0.05 and a power of $80 \%$, we required a sample size of 52 patients. We estimated a $10 \%$ drop-out rate, and thus included 58 patients in our study.

Patient data are presented as mean \pm SD. We compared BIS

Table 2. Correlation Coefficients of BIS Values of Frontal and Mandibular Sensor Positions

\begin{tabular}{lccccccc}
\hline & $\begin{array}{c}\text { Total } \\
(\mathrm{n}=813)\end{array}$ & $\begin{array}{c}\text { Awake } \\
(\mathrm{n}=58)\end{array}$ & $\begin{array}{c}\text { LOC } \\
(\mathrm{n}=58)\end{array}$ & $\begin{array}{c}\text { Intubation } \\
(\mathrm{n}=58)\end{array}$ & $\begin{array}{c}\text { Incision } \\
(\mathrm{n}=58)\end{array}$ & $\begin{array}{c}\text { Maintenance } \\
(\mathrm{n}=465)\end{array}$ & $\begin{array}{c}\text { Emergence } \\
(\mathrm{n}=58)\end{array}$ \\
\hline Correlation coefficient $(\mathrm{R})$ & 0.869 & 0.391 & 0.341 & 0.845 & 0.601 & 0.607 & 0.253 \\
P value & 0.000 & 0.005 & 0.016 & 0.000 & 0.000 & 0.000 & 0.077 \\
\hline
\end{tabular}

Correlation coefficients were calculated by scatter plot analysis. BIS: bispectral index, LOC: loss of consciousness.

A

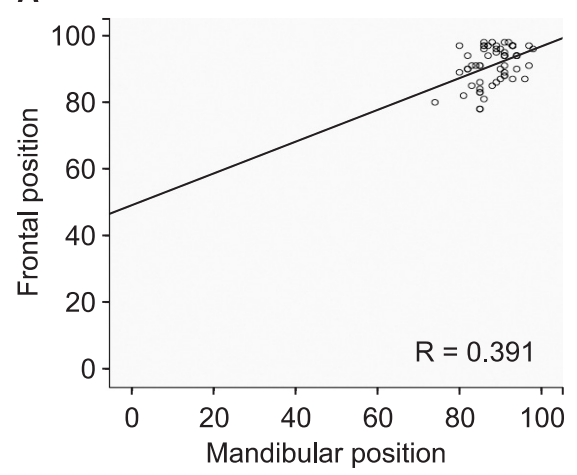

D

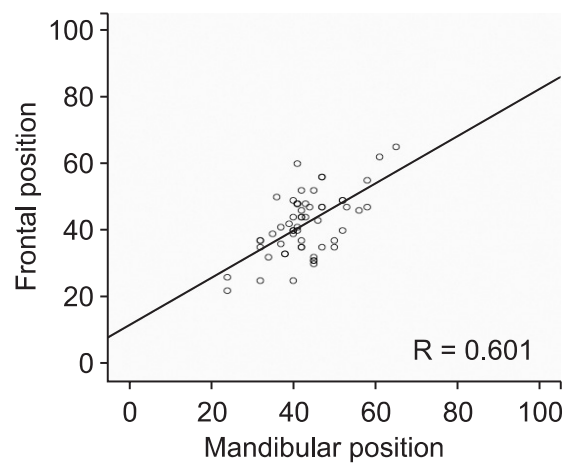

G

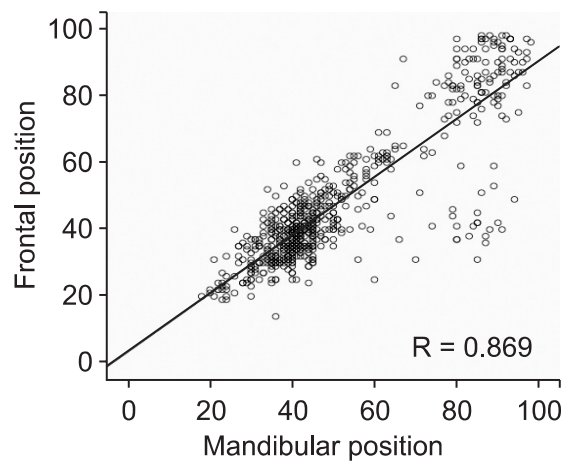

B

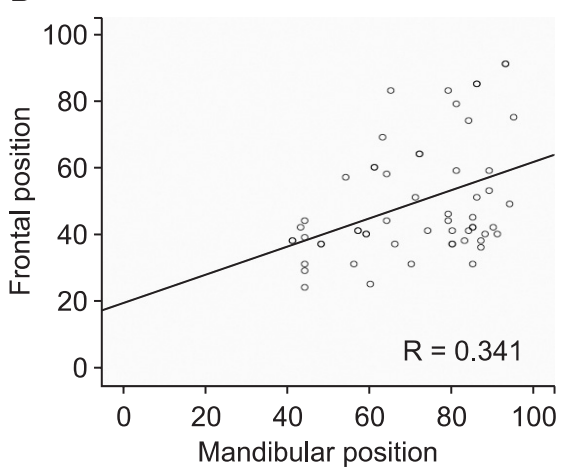

E

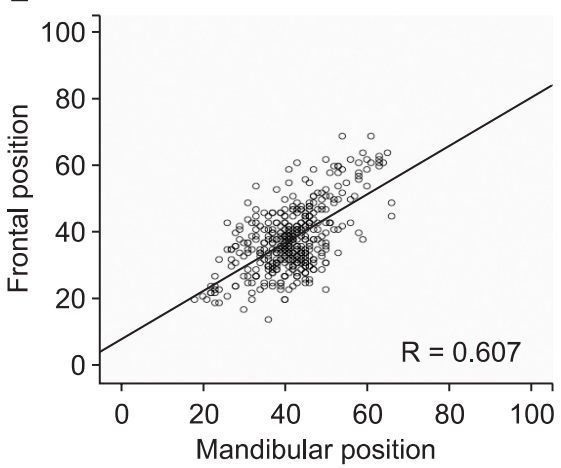

C

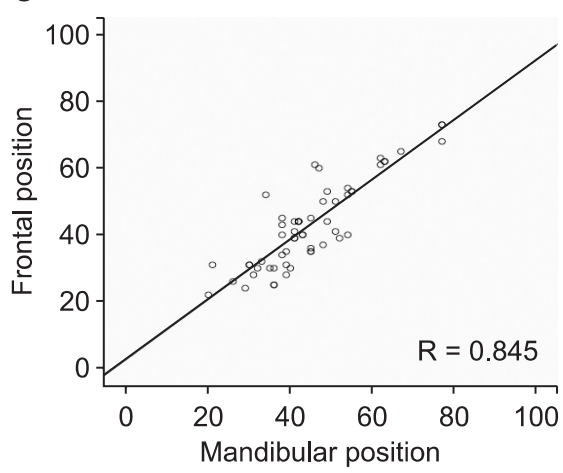

F

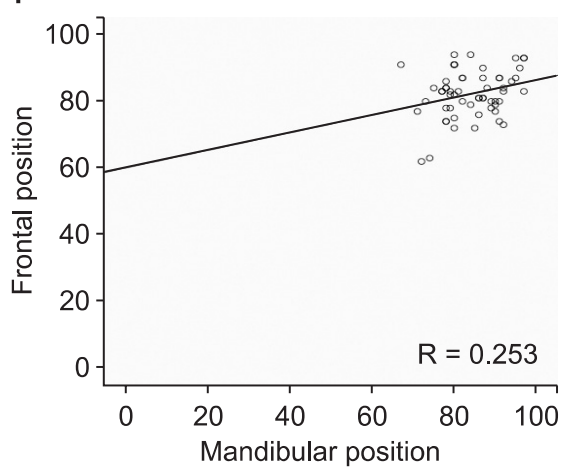

Fig. 2. Scatter plot comparing the standard frontal sensor position and an alternative mandibular position. The straight line represents the trend line for the data set. (A) Awake, (B) Loss of consciousness, (C) Intubation, (D) Incision, (E) Maintenance, (F) Emergence, (G) Overall. 
scores from both sensor positions using linear regression analysis and used Bland-Altman plots to assess agreement between frontal and mandibular sensors with a 95\% confidence interval (CI). The "limit of agreement" was defined as a bias of \pm 1.96 $\mathrm{SD}$, in which $95 \%$ of the difference between the 2 placements were expected to lie between the limits, which would suggest that the 2 positions could be used interchangeably. According to previous studies $[5,6]$, we considered a clinically acceptable level of "limit of agreement" to be \pm 10 BIS units. We used scatter plots to evaluate BIS score correlations and calculated the $\mathrm{R}$ coefficient. We performed paired t-tests to compare differences in EMG and SQI scores. Statistical analysis was performed using

Table 3. Bland-Altman Analysis

\begin{tabular}{|c|c|c|c|c|c|c|c|}
\hline & $\begin{array}{c}\text { Total } \\
(\mathrm{n}=813)\end{array}$ & $\begin{array}{l}\text { Awake } \\
(\mathrm{n}=58)\end{array}$ & $\begin{array}{c}\text { LOC } \\
(n=58)\end{array}$ & $\begin{array}{l}\text { Intubation } \\
\quad(\mathrm{n}=58)\end{array}$ & $\begin{array}{l}\text { Incision } \\
(\mathrm{n}=58)\end{array}$ & $\begin{array}{l}\text { Maintenance } \\
\quad(\mathrm{n}=465)\end{array}$ & $\begin{array}{c}\text { Emergence } \\
\quad(\mathrm{n}=58)\end{array}$ \\
\hline Mean difference & -3.2 & -2.9 & -21.7 & -1.9 & -1.0 & -1.8 & -1.9 \\
\hline $95 \% \mathrm{CI}$ & $16.5 /-22.9$ & $14.1 /-8.3$ & $14.9 /-58.3$ & $11.0 /-14.7$ & $14.2 /-16.2$ & $9.0 /-12.5$ & $14.9 /-18.8$ \\
\hline Outside limit (\%) & 3.7 & 3.4 & 1.7 & 5.0 & 1.7 & 4.3 & 3.4 \\
\hline
\end{tabular}

CI: confidence interval, LOC: loss of consciousness.

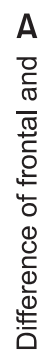

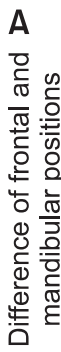
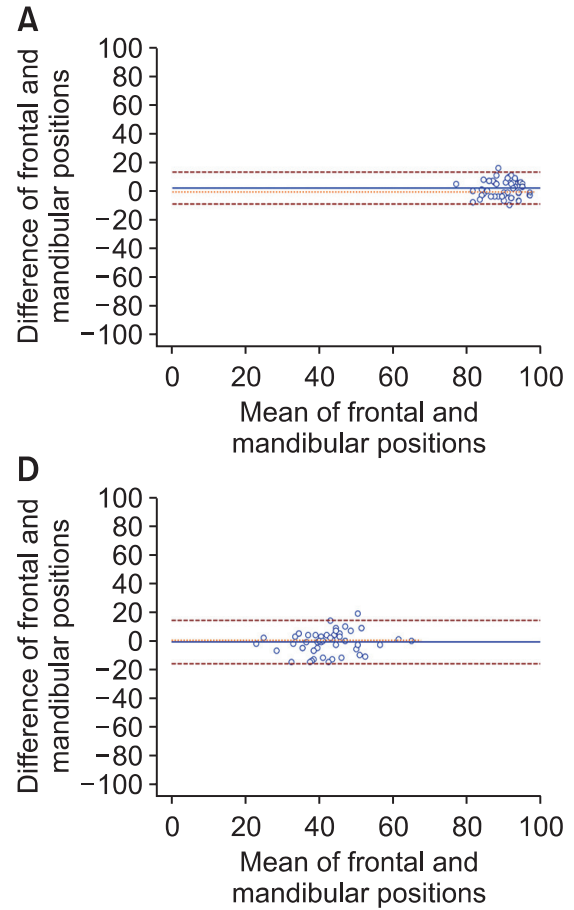

G

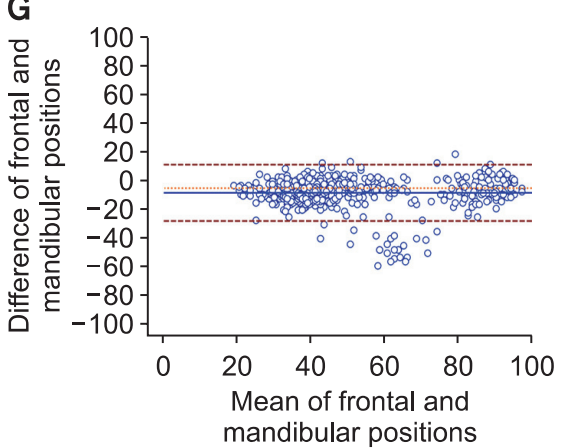

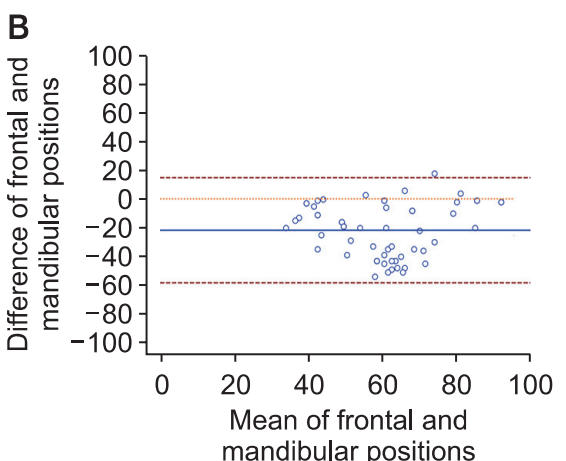
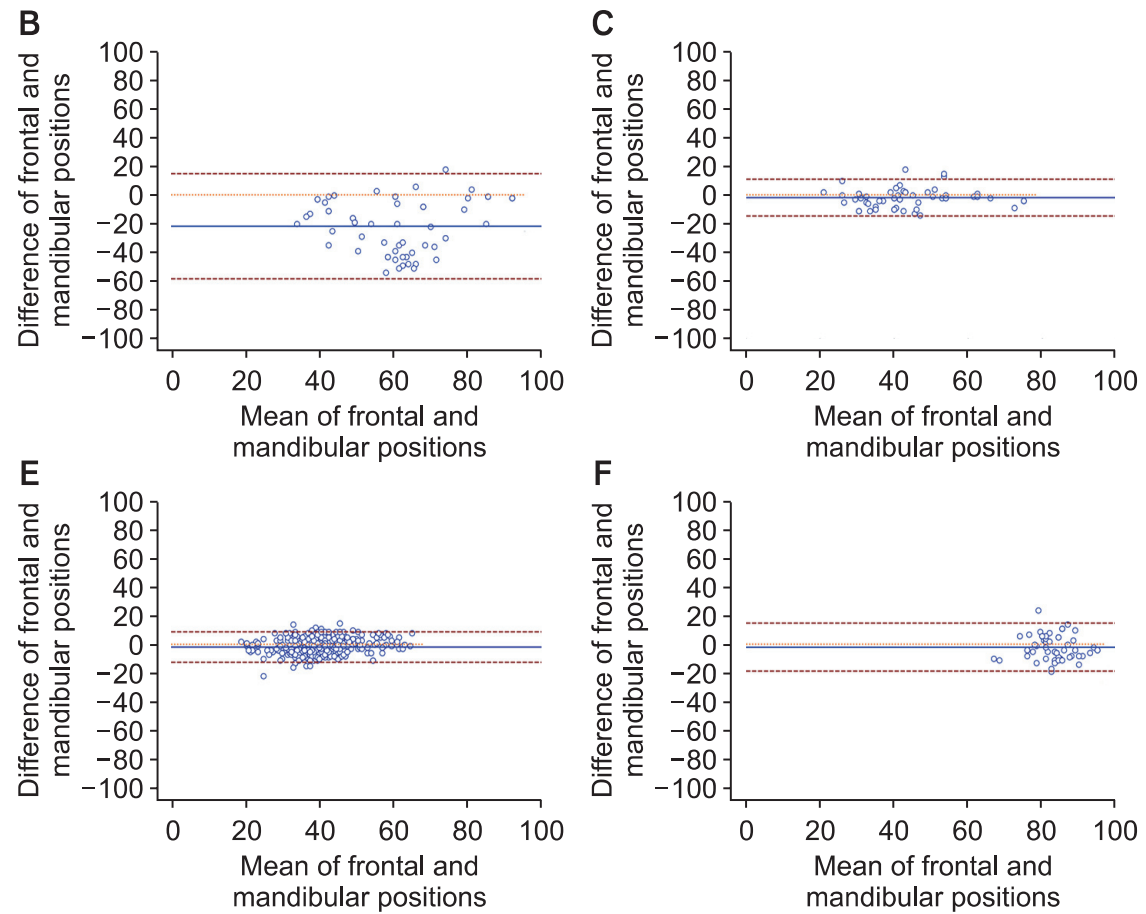

Fig. 3. Bland-Altman scatter plot comparing the standard frontal position with an alternative mandibular position. The solid horizontal line represents the mean difference between frontal and mandibular BIS values. The dotted horizontal lines represent the $95 \%$ limit of agreement between frontal and mandibular BIS values. The $95 \%$ limit of agreement is drawn at the mean difference $+/-1.96$ times the standard deviation of the difference. (A) Awake, (B) Loss of consciousness, (C) Intubation, (D) Incision, (E) Maintenance, (F) Emergence, (G) Overall. 
SPSS software (SPSS version 18.0, IBM, USA) and Medcalc for Windows, version 12.5 (MedCalc Software, Ostend, Belgium). We used a $\mathrm{P}$ value less than 0.05 as the cutoff for statistical significance.

\section{Results}

Patient characteristics are summarized in Table 1. There were significant correlations between frontal and mandibular BIS values at all time points except emergence $(\mathrm{P}=0.077$; Table 2 , Fig. 2). Bland-Altman analysis of frontal and mandibular BIS values resulted in a bias of -3.2 with limits of agreement of 16.5 and -22.9 , respectively. Limits of agreement ranged from 9.0 to -12.5 during maintenance and 14.9 to -58.3 at LOC (Table 3, Fig. 3).

EMG and SQI varied by time point. EMG scores were lowest intraoperatively and highest during emergence. Mean EMG scores were below 40 at all time points except during awake and emergence time points. Mean EMG scores were significantly different between frontal and mandibular positions during the awake, intubation, and emergence time points: mandibular scores were significantly higher than frontal scores at all time points except LOC, incision and maintenance. Average SQI was over 80. In the frontal position, mean SQI scores were below 80 during awake or intubation time points, while in the mandibular position, they were below 80 during awake, LOC, intubation and emergence time points. Conversely, mean SQI scores were significantly higher in the frontal than in the mandibular position except during incision and maintenance time points (Table 4).

The time it took for BIS scores to fall to the lowest level during induction varied greatly between frontal and mandibular positions (range $0-120 \mathrm{sec}$, mean $\pm \mathrm{SD}=26.9 \pm 27.4 \mathrm{sec}$ ).

\section{Discussion}

Our research shows that BIS, EMG, and SQI scores from the 2 sensor positions vary by anesthesia time point. The correlation coefficient between the 2 positions indicated a strong correlation during intubation $(\mathrm{R}=0.845)$, incision $(\mathrm{R}=0.601)$, and the intraoperative maintenance period $(\mathrm{R}=0.607)$, as well as a weak correlation during awake $(\mathrm{R}=0.391)$ and LOC $(\mathrm{R}=0.341)$ time points. During the emergence time point, there was no correlation between BIS values from the frontal and mandibular positions. We hypothesize that this pattern of correlation is related to physiologic changes that occur in different anesthesia stages: during a stable sedation state with sufficient muscle relaxation and low BIS scores, the correlation coefficient is higher than in other stages.

Bland Altman analysis also reveals similar findings. The mean difference was lowest at incision $(-1.0)$ and highest at loss of consciousness $(-21.7)$, and was relatively lower at intubation $(-1.9)$ and during maintenance $(-1.8)$. The percentage of BIS scores outside of the limit of agreement is only $3.7 \%$ overall, and not over $5 \%$ in any anesthetic stage. However, there is variability in $95 \%$ CI depending on various anesthetic states. Although a clinically acceptable limit of agreement has not been defined, some authors consider it to be \pm 10 BIS units $[5,6]$. In our case, 95\% CI is over 10 BIS units. However, in our study, we found a relatively narrow limit of agreement during stable states (awake, $14.1 /-8.3$; and maintenance, $9.0 /-12.5$ ) by comparison with the other anesthetic states that were expected a relatively wider change in BIS score (LOC, 14.9/-58.3, and emergence, 14.9/-18.8). These data suggest that sudden changes in BIS score reduce the reliability of mandibular sensor placement.

Overall, BIS values do not agree between the standard frontal position and the alternative mandibular position. But correlation coefficient between two sensor position showed strong relationship $(\mathrm{R}=0.607)$ and Bland Altman analysis revealed low mean difference $(-1.8)$ and relatively low limit of agreement (9.0/12.5 ) with only $4.3 \%$ of the values lying outside of the limit of agreement during anesthetic maintenance period. On the basis of the clinical values and correlation, it can be clinically acceptable. Therefore, during the anesthesia maintenance period, the mandibular position can be availably used as an alternative position if the operative field renders the standard position unavailable.

EMG scores varied greatly depending on anesthetic state. The difference in mean EMG score was statistically significant

Table 4. Comparison of EMG and SQI Scores

\begin{tabular}{|c|c|c|c|c|c|c|c|c|}
\hline & & $\begin{array}{c}\text { Total } \\
(\mathrm{n}=813)\end{array}$ & $\begin{array}{l}\text { Awake } \\
(\mathrm{n}=58)\end{array}$ & $\begin{array}{c}\text { LOC } \\
(\mathrm{n}=58)\end{array}$ & $\begin{array}{l}\text { Intubation } \\
(\mathrm{n}=58)\end{array}$ & $\begin{array}{l}\text { Incision } \\
(\mathrm{n}=58)\end{array}$ & $\begin{array}{l}\text { Maintenance } \\
\quad(\mathrm{n}=465)\end{array}$ & $\begin{array}{l}\text { Emergence } \\
(\mathrm{n}=58)\end{array}$ \\
\hline \multirow{2}{*}{ EMG } & Frontal & $33.1 \pm 11.3$ & $47.6 \pm 7.2$ & $36.4 \pm 12.4$ & $30.3 \pm 4.4$ & $29.7 \pm 10.5$ & $27.8 \pm 6.0$ & $50.8 \pm 9.7$ \\
\hline & Mandibular & $35.1 \pm 14.1^{*}$ & $54.2 \pm 7.4^{*}$ & $40.0 \pm 11.0$ & $34.9 \pm 10.3^{*}$ & $28.8 \pm 9.7$ & $28.3 \pm 8.8$ & $55.8 \pm 10.8^{*}$ \\
\hline \multirow[t]{2}{*}{ SQI } & Frontal & $85.2 \pm 15.3$ & $68.5 \pm 16.3$ & $80.9 \pm 12.8$ & $76.4 \pm 12.0$ & $86.0 \pm 17.6$ & $90.6 \pm 12.4$ & $80.3 \pm 14.1$ \\
\hline & Mandibular & $80.9 \pm 18.8^{*}$ & $58.3 \pm 15.8^{*}$ & $73.1 \pm 15.8^{*}$ & $67.0 \pm 14.4^{*}$ & $84.5 \pm 15.7$ & $89.5 \pm 14.1$ & $66.7 \pm 17.4^{*}$ \\
\hline
\end{tabular}

Data are presented as mean \pm SD. EMG: electromyography, SQI: signal quality index, LOC: loss of consciousness. *P $<0.05$ compared with frontal sensor position. 
between frontal and mandibular sensor positions during the awake and emergence time points. This may be due to increased muscle tone during these stages. Overall, the mean EMG score was higher in the mandibular than in the frontal position. This may be due to more muscle movement in the chin than in the forehead, since this difference became statistically insignificant after administration of the muscle relaxant. The significant difference between mean EMG scores for the 2 sensor positions during intubation was likely due to the jaw manipulation required for endotracheal intubation although sufficient muscle relaxation enough to intubation was exist. Facial muscle EMG activity can produce artifact in the EEG, greatly impacting the BIS score [7]. Higher EMG in mandible sensor could influence not only relatively higher BIS values of mandible sensor than that of frontal sensor but also negative aspect of equivalent of two sensors. Consistent with this, overall, BIS scores from the mandibular sensor position were higher than those from the frontal position.

Mean SQI scores were relatively lower in the mandibular than in the frontal sensor position. However, during incision and maintenance periods, mean SQI was over 80, and without statistically significant difference, in both sensor positions. SQI measures the quality of the acquired EEG signal (0-100\%). SQI is more than 80 , it ensured good quality data [8]. This result indicates the reliability of the mandibular sensor position during the stable anesthetic state. Conversely, mean SQI was significantly higher in the frontal than in the mandibular sensor position during awake, LOC, intubation, and emergence time points, indicating low clinical accuracy of the mandibular position. Other factors such as a strong carotid pulse, use of the upper body forced air warming units or electrocardiography can affect the BIS scores of mandibular sensor as an artifact. And these interferences can lead to false interpretation of BIS readings without any sign of artifact recognition $[9,10]$.

After intravenous anesthetics were given, BIS values fell acutely at the LOC time point. In our previous pilot study, we found the frontal BIS values fell more quickly than mandibular BIS values during induction. The time it took for BIS scores to fall to the lowest level during induction varied greatly between frontal and mandibular positions (range, 0-120 sec). This finding suggests that the spread of EEG current to the chin may delay conduction time.

Other authors have proposed that BIS score is a topographic dependent variable in light of the heterogeneous EEG findings in BIS sensors placed on non-frontal areas. One study on occipital placement showed a +10 BIS score bias under deep anesthesia and a -10 BIS bias before induction. Although the nature of the BIS algorithm is not disclosed by the creators of this technology, this result may be due to the predominance of posterior alpha $(\alpha)$ waves in the awake brain and generation of delta $(\delta)$ and theta $(\theta)$ activity under deep propofol anesthesia [5]. In contrast, strong correlations have been reported between frontal and occipital sensor placement, as well as between fronto-central and bifrontal placement $[4,11]$. Unlike the frontal or occipital area, no EEG is generated under the mandible, and thus detectable EEG or BIS is likely conducted from other parts of cerebral cortex. The exact locations remain unknown and warrant further study.

There are several limitations to our study. First, our study did not standardize the anesthetic methods used, which may have influenced the results [12]. Second, BIS-vista does not generate raw EEG tracing for analysis, we were unable to confirm that the actual EEG recordings were identical. Although the BIS algorithm has not been formally validated, actual EEG recordings at each electrode on frontal and mandibular positions could help to confirm the accuracy and characteristics of the EEG signal arising from the mandible.

In conclusion, during the anesthesia maintenance period, the mandibular position can be availably used as an alternative position if the operative field renders the standard position unavailable.

\section{References}

1. Kearse LA Jr, Manberg P, Chamoun N, deBros F, Zaslavsky A. Bispectral analysis of the electroencephalogram correlates with patient movement to skin incision during propofol/nitrous oxide anesthesia. Anesthesiology 1994; 81: 1365-70.

2. Liu J, Singh H, White PF. Electroencephalographic bispectral index correlates with intraoperative recall and depth of propofol-induced sedation. Anesth Analg 1997; 84: 185-9.

3. Sigl JC, Chamoun NG. An introduction to bispectral analysis for the electroencephalogram. J Clin Monit 1994; 10: 392-404.

4. Shiraishi T, Uchino H, Sagara T, Ishii N. A comparison of frontal and occipital bispectral index values obtained during neurosurgical procedures. Anesth Analg 2004; 98: 1773-5.

5. Dahaba AA, Xue JX, Zhao GG, Liu QH, Xu GX, Bornemann H, et al. BIS-vista occipital montage in patients undergoing neurosurgical procedures during propofol-remifentanil anesthesia. Anesthesiology 2010; 112: 645-51.

6. Nelson P, Nelson JA, Chen AJ, Kofke WA. An alternative position for the BIS-Vista montage in frontal approach neurosurgical cases. J Neurosurg Anesthesiol 2013; 25: 135-42.

7. Bruhn J, Bouillon TW, Shafer SL. Electromyographic activity falsely elevates the bispectral index. Anesthesiology 2000; 92: 1485-7. 
8. Sinha PK, Koshy T. Monitoring Devices for Measuring the Depth of Anaesthesia - An Overview. Indian J Anaesth 2007; 51: 365-81.

9. Hemmerling TM, Charabati S, Bracco D. Unusual case of low bispectral index values due to electrocardiographic interferences. Br J Anaesth 2008; $101: 877-8$.

10. Hemmerling TM, Fortier JD. Falsely increased bispectral index values in a series of patients undergoing cardiac surgery using forced-airwarming therapy of the head. Anesth Analg 2002; 95: 322-3.

11. Glass PS, Bloom M, Kearse L, Rosow C, Sebel P, Manberg P. Bispectral analysis measures sedation and memory effects of propofol, midazolam, isoflurane, and alfentanil in healthy volunteers. Anesthesiology 1997; 86: 836-47.

12. Dahaba AA. Different conditions that could result in the bispectral index indicating an incorrect hypnotic state. Anesth Analg 2005; 101: 765-73. 